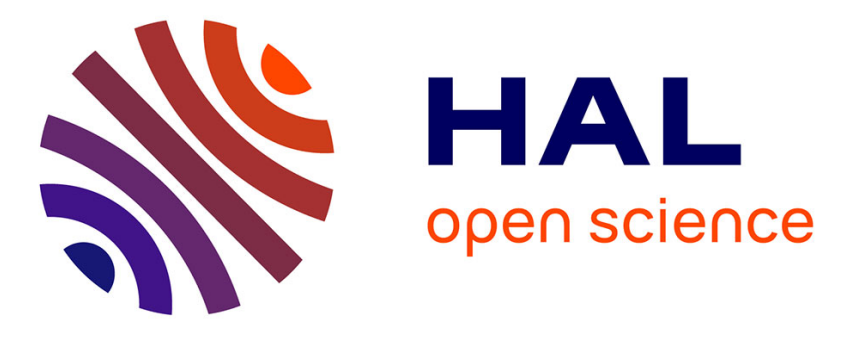

\title{
Designing Smart Adaptive Flooding in MANET using Evolutionary Algorithm
}

\author{
Wahabou Abdou, Christelle Bloch, Damien Charlet, Dominique Dhoutaut, \\ François Spies
}

\section{- To cite this version:}

Wahabou Abdou, Christelle Bloch, Damien Charlet, Dominique Dhoutaut, François Spies. Designing Smart Adaptive Flooding in MANET using Evolutionary Algorithm. MobilWare 2011, 4th Int. ICST Conf. on MOBILe Wireless MiddleWARE, Operating Systems, and Applications, Jan 2011, United Kingdom. pp.71 - 84. hal-00943167

\section{HAL Id: hal-00943167 https://hal.science/hal-00943167}

Submitted on 7 Feb 2014

HAL is a multi-disciplinary open access archive for the deposit and dissemination of scientific research documents, whether they are published or not. The documents may come from teaching and research institutions in France or abroad, or from public or private research centers.
L'archive ouverte pluridisciplinaire HAL, est destinée au dépôt et à la diffusion de documents scientifiques de niveau recherche, publiés ou non, émanant des établissements d'enseignement et de recherche français ou étrangers, des laboratoires publics ou privés. 


\title{
Designing Smart Adaptive Flooding in MANET using Evolutionary Algorithm
}

\author{
Wahabou Abdou, Christelle Bloch, Damien Charlet, \\ Dominique Dhoutaut, and François Spies \\ Computer Science Laboratory of the University of Franche-Comté, \\ 1, Cours Leprince-Ringuet 25201 Montbéliard - France \\ firstname.lastname@univ-fcomte.fr,
}

\begin{abstract}
This paper deals with broadcasting security messages in mobile ad hoc networks (MANETs). Traditional broadcasting schemes tend to focus on usually high and homogeneous neighborhood densities environments. This paper presents a broadcasting protocol that locally and dynamically adapts its strategy to the widest range of neighborhood densities. The behavior of the protocol is tuned using various internal parameters. Multiple combinations of those parameters have been precomputed as optimal solutions for a range of neighborhood densities, and the most relevant one is dynamically chosen depending on the locally perceived environment. The combinations were determined by coupling an evolutionary algorithm and a network simulator (ns-2), using a statistically realistic radio-propagation model (Shadowing Pattern). This approach is compared with other probabilistic methods while broadcasting an emergency message in Vehicular Ad hoc Networks (VANETs) with variable and heterogeneous vehicle densities. In such a context, it is expected from the network to enable each of the nodes to receive the warning message. The results show that our protocol covers the whole network, whereas other methods only reach between $57 \%$ and $90 \%$ coverage.
\end{abstract}

Key words: MANET; VANET; Flooding; Broadcast Storm Problem; Evolutionary Algorithm

\section{Introduction}

The broadcast is a regularly used mode of communication in Mobile Ad hoc Networks (MANETs). It is used by routing protocols for route discovery and maintenance. In such networks, a wide spread of packets is possible only if they are relayed by some nodes. However, nodes share the wireless channel and an inappropriate relay strategy can lead to a channel saturation or packets losses and prevent a wide dissemination. The diffusion scheme depends on the network density. Several broadcasting methods have been proposed for MANETs to optimize the channel use. Notably, probabilistic methods determine the probability $P$ to retransmit a packet for every node, and each packet is retransmitted at 
most once per node. These solutions have reduced the number of redundant packets.

In general, existing solutions have not been designed for very low densities networks. This paper proposes to add new parameters to probabilistic broadcasting methods to adapt the diffusion strategy to various network densities. Accordingly, it expands the search space and the complexity of the problem. Therefore an evolutionary algorithm is used to determine the parameter combinations that best fit to various levels of the network density. A network simulator (ns-2) is used to assess the dissemination of packets using each parameter combination. The broadcast protocol resulting from this proposal is then compared to three other probabilistic methods. Initially, comparisons were made for networks with homogeneous densities, then for a heterogeneous network.

The remainder of this paper is organized as follows. Section 2 presents an overview of MANETs broadcasting methods. Section 3 describes the proposed broadcasting protocol which adapts its dissemination strategy to the network density. The experiments for both homogeneous and heterogeneous networks are outlined in Section 4. Section 5 presents concluding remarks and outlines future work.

\section{Broadcasting methods in ad ho networks}

Message broadcasting in MANETs consists in sending a packet from one node to every node within its transmission range. This kind of communication is a recurring task which is specially used by routing protocols for route discovery. It is worthy of note that in networks such as WiFi, a node cannot both send and receive packets at the same time. Such a situation would cause collisions and generate errors on both communications (transmission and reception). To reduce the risk of interference and thus optimize the use of the radio channel, several broadcasting methods have been proposed for MANETs and some of them are dedicated to delay tolerant networks.

\subsection{Generic methods for MANETs}

The broadcasting methods could be classified with respect to the nature of the algorithms: deterministic or non-deterministic.

Deterministic methods are methods whose behaviors are (quasi-)predictable, and whose decisions are not based on random variables ${ }^{1}$. This group, gathers simple flooding, neighbor-knowledge approaches and multi-point relay methods.

Simple flooding: is obviously the simplest broadcasting strategy. Each node relays received packets exactly once. Duplicate messages are discarded. This method does not take into account the neighborhood density of nodes. In high

\footnotetext{
${ }^{1}$ This notion of determinism concerns only the decisions of the broadcast but not the channel access (layer 2) which may be based on random methods.
} 
density networks, the simple flooding wastes the bandwidth and may leads to network contention.

Neighbor knowledge-based methods: using "Hello" packets, nodes build a 1hop or 2-hop neighbors lists. These lists are suffixed to the broadcast packets so that the receiver $(r)$ can compare the sender's list to its own list.This comparison determines the additional nodes that will receive the message if $r$ forwards it. For static or low mobility networks, it is a fair method. But when the node's velocity is high, the information about the neighbors become quickly inaccurate. Alba et al. [1] have proposed an improvement of the knowledge-based methods. The authors used a cellular multi-objective genetic algorithm to find the values of the parameters that help to assess whether to forward a message.

Multi-point relay (MPR): it is a variation of knowledge-based techniques suggested by the Hypercom team of INRIA ${ }^{2}$ lab. To reduce the number of redundant broadcasts of a packet in the network, each node chooses several nodes among its neighbors that will relay its communications. The selected nodes are called MPRs [2]. When a node sends a packet on the radio channel, all its neighbors will receive it, but only the MPRs of the source node will relay the message. That means each node will have a list of all nodes that have chosen it as their "repeater" (MPRs selectors list). The MPRs are selected among the 1-hop neighbors so that they enable the node that has chosen them to reach all its 2-hop neighbors. The goal is to have the smallest list of MPRs in the network which optimizes communications. The MPRs require a bidirectional link.

\section{Non-deterministic methods}

Probabilistic methods: they aim to improve the simple flooding method. Upon the reception of a packet, the node forwards or discards it depending on a given probability $p$ [3] [4] [5]. A challenge is to set the value of $P$. Although Li et al. [6] suggest that values between 0.6 and 0.8 are optimal, it is obvious that they are not optimal for all network densities. If $P=1$, this method is equivalent to simple flooding.

Counter-based schemes: they require nodes to count the number of the redundant copies of a single message over a short period of time called Random Access Delay (RAD) [7]. When the RAD expires, if the number of copies is less than a given threshold $\left(c_{t}\right)$, the message is forwarded. Otherwise, it is dropped. This method implies additional latency.

Location-based methods: before relaying a message, the node evaluates the additional coverage area that will result from this retransmission. This technique does not consider whether nodes exist within that additional area or not. To evaluate the extra coverage area, the node can use the distance between itself and each node that has previously relayed the message (distance-based scheme) or the geographical coordinates (location-based scheme). In both distance-based and location-based schemes, a RAD is assigned before the message is relayed (if the additional coverage area is higher than a fixed threshold) or dropped.

\footnotetext{
${ }^{2}$ Institut National de Recherche en Informatique et en Automatique, France
} 


\subsection{Diffusion methods dedicated to delay tolerant networks}

In a sparse environment, communications in VANETs behave like those studied by the Delay/Disruption Tolerant Network (DTN) community. Thus, flooding protocols developed for DTN might be used in VANETs. The epidemic routing [8] scheme proposes that a node relays the message to all the nodes it crosses, which did not know about it. This is a monotone relay strategy. The PRoPHET protocol [9] (Probabilistic Routing Protocol using History of Encounters and Transitivity) applies probabilistic routing instead of doing blind epidemic replication of bundles through the network. it uses a metric called 'delivery predictability' established at every node A for each known destination B.

-Because of the low reception rate found in such environments, most of these protocols schedule multiple transfer of the same message. The number of replica may range from one copy (e.g., direct transmission protocol [10]) to an infinite as in epidemic routing. As it has a direct impact on the network load, much effort has been undertaken to leverage the cost of forwarding by finding the most valuable tradeoff between cost and reliability [11]. It should be noted that most of the advanced scheme are tuned for sparse environments, and do not scale to be used in medium or high density environments.

\section{A new neighborhood density-aware method}

\subsection{Challenges}

One of the main challenges of broadcasting problems in wireless ad hoc networks is to reach the maximum number of nodes while avoiding useless repeats. Recent work in this field shows the need to reduce the number of relay nodes when the network density is high. However, as mentioned in Section 2, a part of those improvements is to the detriment of the delay, especially because a waiting time (RAD) is added. Another weakness of existing methods is the impracticability of their proposals in very sparse networks (i.e. the probability that a node has a neighbor is very low) and environments where the broadcast packets may be lost. Indeed, according to the WiFi standard, broadcast packets are not acknowledged. Thus, the source node cannot be sure that the packets are received. To solve this problem, it is necessary to retransmit packets more than once in certain cases. In our proposal, we suggest to improve the existing probabilistic methods by adding new parameters. We use the following four parameters to regulate broadcast in MANETs:

- The probability $(P)$ to relay a packet. Upon the reception of a packet, each node decides to forward or to drop it depending on the value of $P$. This is the main parameter of the probabilistic methods.

- The number of times each packet will be repeated $(\mathrm{Nr})$. In the case of lowdensity mobile networks, a node may not have a neighbor in its coverage area when it forwards a packet. By repeating the packets more than once, this node increases its chance of being heard by another mobile node. This parameter 
can also be useful when the initial transmission is lost due to collisions or other phenomena related to radio propagation conditions.

- The delay $(D r)$ between two successive repeats. Indeed, if a node has to repeat the packets several times, one must determine the frequency at which the redundant copies should be sent. Please note that in a dense network, low values of $D r$ could lead to the increase of the number of collisions.

- The TTL (Time To Live). This parameter permits to confine the spread of messages in a given geographic area. It specifies the number of hops allowed to the packets.

The variation range of these parameters is given in Table 1 .

Using these four parameters, the solutions are simulated using the Network Simulator 2 (ns-2) [12]. As the simulations are stochastic processes, each solution is evaluated by the simulator 500 times $^{3}$ to obtain statistically reliable results. ns- 2 assesses the solutions using the four following criteria:

\begin{tabular}{|l|l|l|l|l|}
\hline Parameter & $P$ & $N r$ & $D r$ (in seconds) & $T T L$ \\
\hline Lower bound & 0 & 1 & 0 & 10 \\
\hline Upper bound & 1 & 30 & 2 & 40 \\
\hline
\end{tabular}

Table 1: Variation ranges of decision variables

- NC: the average number of collisions;

- PT: the average propagation time (the time spent until all the nodes in the considered area receive the message);

- $R$ : the average number of retransmissions during the simulation.

- FR: the full reception ratio. It is the ratio between the number of successful simulations ${ }^{4}$ and the total number of simulations (500 in our experiments).

Relying on these parameters and criteria, our method is based on three steps:

- Simulating ad hoc networks with different neighborhood densities to determine the appropriate values of $P, N r, D r$ and $T T L$ in every context;

- Enabling the nodes to determine their neighborhood density (without sending HELLO packets, or adding a waiting time);

- Making the nodes able to automatically change their broadcasting strategy by choosing the one that best suits the environment of each node.

The following subsections detail these three steps.

3 This value is determined empirically. It represents a good compromise between the result confidence interval and the simulation time

${ }^{4}$ For each of the 500 simulations, if the channel is saturated, the number of collisions may prevent the message from being normally transmitted to all the nodes. In very low density networks, communications between nodes may also break or deteriorate gradually and prevent a complete reception by the nodes. In these cases, the simulation is stored as a failed simulation. 


\subsection{Setting broadcasting parameters for various densities}

The variation ranges given in Table 1 result in a search space that contains $9.10^{16}$ possible combinations. Running each combination requires from a dozen seconds up to a few minutes according to the considered neighborhood density. Simulating all possible cases with ns-2 would take a long time. That is why an evolutionary algorithm (EA), inspired by the theory of natural evolution, is used to assess a subset of interesting solutions. Figure 1 illustrates the three main modules of the proposed approach (an optimization engine, a network simulator and a $\log$ analyzer) and their interaction.

Firstly, the EA randomly generates a set of $n$ possible solutions called initial population. (In Figure 1 the " $P_{0}$ ?" condition is used to check if the current population is the initial one.) Each solution, i.e. a set of possible parameters, must be evaluated and fitness is assigned to it. In our case, the evaluation process is done in two steps: the first is performed by the "Network simulator" and "Log analyzer" modules; the second is assessed by the EA. Firstly, each set of parameters is transmitted to the network simulator. The latter integrates the received parameters into the simulation scripts. Thereafter, the simulations are run and some log files are built. These files describe the network behavior. The log files are passed on to the log analyzer that extracts the values of the objective functions. The calculated objective values are then conveyed to the optimization engine that ranks the solutions according to these values, using the concept of Pareto dominance. Pareto solutions are those for which improvement in one objective implies the worsening of at least one other objective. Four Pareto fronts are built $\left(R_{1}, R_{2}, R_{3}\right.$ and $R_{4} . R_{4}$ represents the dominated solution list). Thereafter two individuals (called parents) are selected for recombination.Each parent is chosen by two random selections. The Pareto front is first selected according to a computed probability. The probability to select each list both takes into account a priority level associated to each list's and the list length. This favours the best solutions while preventing the dominated solutions from having very low values of fitness, in order to preserve the diversity of the successive populations. Then an individual is randomly selected among the individuals belonging to this front, using equal probabilities for all these individuals. Each pair of selected parents is recombined using a simulated binary 10-point crossover (variables are first converted into binary strings). The k-point crossover operator was chosen because it is a classical method of recombination. The value of $\mathrm{k}$ was empirically tuned. Finally, a uniform mutation is applied: a gene is randomly chosen and the EA generates a new value for this variable with respect to its variation range. These operators permit to generate a list of offspring, whose fitness is again computed using ns- 2 simulations. Each offspring replaces the first parent it dominates in the population list. If the offspring does not dominate any parent, it is not added to the list of individuals of the next generation. All these steps (evaluation, selection, crossover, mutation, replacement) are repeated until a given stop criterion is met. The EA finally returns an archive of $R_{1}$ built over generations. 


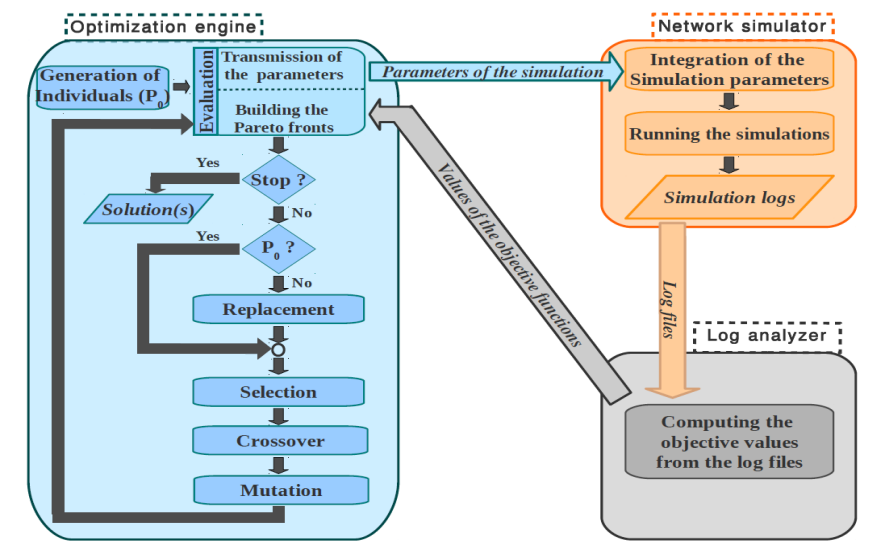

Fig. 1: Flow chart of the optimization tool

The evolutionary algorithm used is detailed in [13] where we presented our first results in a smaller homogeneous network based on a less in depth search space.

\subsection{Detection of the neighborhood density}

Through communications in the network, each node builds a local view of its neighborhood. This view is mainly based on the number of different neighbors repeating the same packet. To this end, each node keeps a history of the number of neighbors it overhear repeating each packet. Whenever a node receives a packet for the first time, it saves the packet's identifier and set the number of sources for this packet to 1 . This number is incremented each time a copy of this packet is received for the first time from a neighbor. This allows to observe the evolution of communications over time. It should be noted that multiple repetitions by a single source do not increment the number of sources. In order to both save the mobile nodes memory and keep information up-to-date, the packets sources history has limited size and older information are discarded.

The actual number of neighbors, denoted $\overline{n g h}$ is an average computed from the number of neighbors that sent the packets received in the recent history.

In this paper each node uses a simple and passive algorithm to evaluate the actual number of neighbors. As we focus on applications in which information messages are generated regularly, we can manage without active "hello" messages.

\subsection{Choice of the dissemination strategy}

For the implementation of the Smart-flooding, several density levels have been studied using the optimization approach described in Section 3.2. An average 
number of neighbors corresponds to every neighborhood density. For each density level a combination of input parameters $(P, N r, D r$ and $T T L)$ is proposed by the optimization tool. The nodes have a matching table between the network density (number of neighbors) and the strategy to use (values of the input parameters). After each update of $\overline{n g h}$, each node chooses the dissemination strategy which is the most appropriate for its environment.

However when the packet reception history is empty, the node has no information about the network density. Thus, it intends to send packets it should use a special strategy called "initialization strategy". The latter consists of:

1. sending the packet;

2. waiting for a time period $t$ and then controlling the average number of neighbors $(\overline{n g h})$

3. adjusting the communication strategy based on $\overline{n g h}$

4. waiting for a time period $t$ then refining the dissemination strategy depending on the new value of $\overline{n g h}$

\section{Experiments}

\subsection{Validation of the EA}

The Elitist Simulated Binary Evolutionary Algorithm (ESBEA) [14] is used for the following experiments. ESBEA is first compared with three EAs found in literature: two well-known EAs (NSGA-II [15] and SPEA2 [16]) and a more recent one (DECMOSA-SQP [17]). The four algorithms are compared using the CEC 2009 competition procedure on two constrained multi-objective problems proposed in this competition (constrained problems 1 \& 2) [18]. As shown by the results presented in [14], ESBEA provides positive gains for the comparison when using the Inverted Generational Distance (IGD) metrics recommended in CEC 2009 competition.

\subsection{Experimental procedure}

The proposed broadcasting protocol is compared with Simple Flooding (which is known as the reference method for broadcasting problems in MANETs) and two probabilistic methods with probability values respectively equal to 0.6 and 0.8 . These four broadcasting methods are initially evaluated in homogeneous density networks. The simulated networks are Vehicular Ad hoc Networks (VANETs).

VANETs can enhance safety on the roads by communicating traffic information, accidents, bypass, etc. In the remainder of this section, we evaluate the behavior of the broadcasting methods when a vehicle sends a warning message. It is obvious that, under such circumstances, it is essential to spread the message as quickly as possible and most importantly, the message must reach as many vehicles as possible in the vicinity of the transmitter. The experiments 
were carried out using the ns-2 network simulator (ns-2.34). We had to be careful concerning the simulation models because the radio environment has a very strong impact on communications, especially in the very hostile VANET context. We chose to use the Shadowing Pattern [19] propagation model which is a realistic and probabilistic model based on outside monitored communications and it is well suited for our needs. It can produce particularly realistic statistical errors distributions, but it is still computationally easy enough to be carried out on medium to large simulations. Radio communications can be impacted among others by the topography, the buildings, the cars and vehicles passing by, the presence of trees, other radio communications, antenna design and even the weather. Shadowing Pattern does not aim at exhaustively simulating all the complex occurring phenomena. Instead it makes use of experimental calibration to exhibit a global statistical behavior much closer to reality than that of a more realistic but partial model.

-We conducted experiments and made use of Vanet Data Representation (VDR), a software we developed in order to make the analysis of the massive amount of real data collected easier. As shown on Figure 2, VDR displays on a single window various configurable metrics along with the context presented as a dynamic map and even video when available.

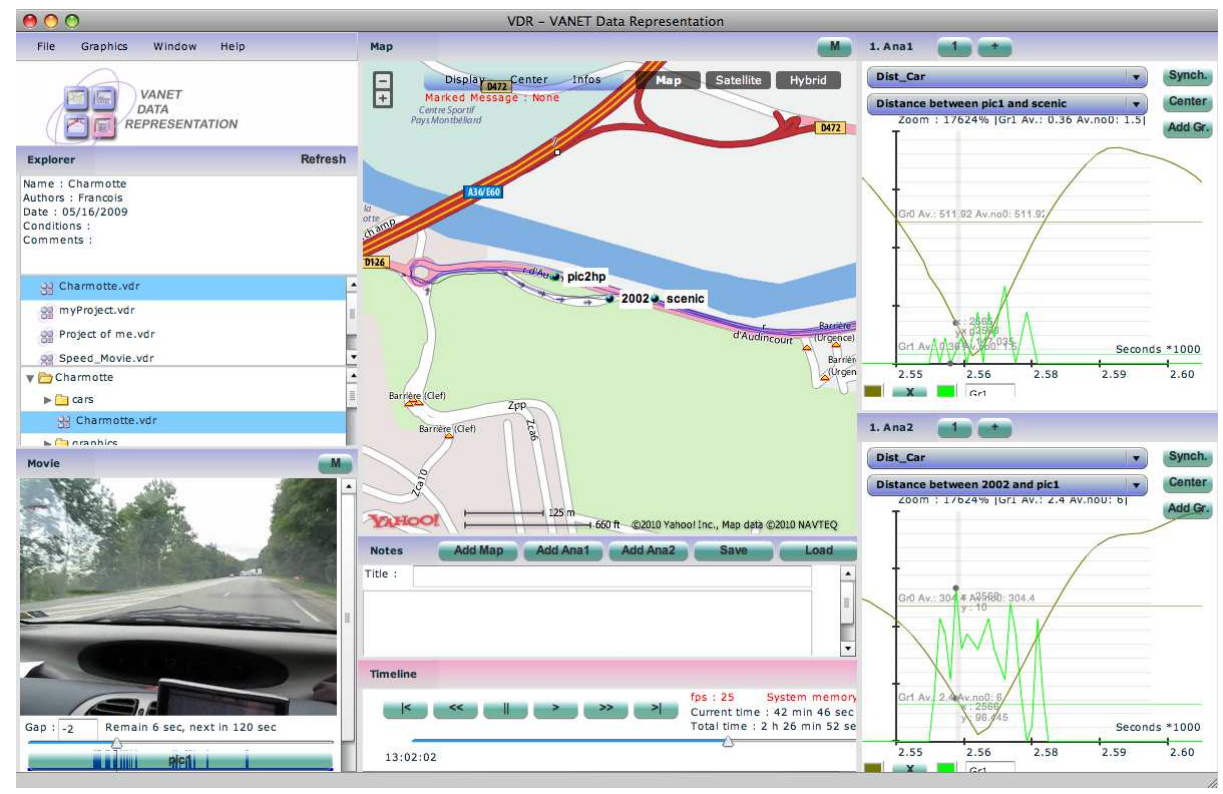

Fig. 2: Vanet Data Representation (VDR) - a tool to analyse real world experiments and to calibrate the Shadowing Pattern radio propagation model 


\subsection{Tests in homogeneous networks}

For experiments in a homogeneous context, we considered four levels of neighborhood densities. For each network, we built a chain topology that illustrates cars lined up on $10 \mathrm{~km}$. We varied the distance between two consecutive vehicles to regulate the density as mentioned in Table 2 . In medium density context, each message may be received by tenths of nodes. Low density topology is similar to a highway network: each vehicle is able to communicate regularly with a dozen peers, but more occasionally packets are received by vehicles up to a few thousand meters away (as observed in real experiments).

The fourth considered density level (very low-density network) represents a collection of vehicles in a rural area where traffic generally flows steadily. A vehicle might have no neighbor in its coverage area. To simulate such a sparse vehicule distribution, the Shadowing Pattern propagation model has been set so that it mimics the very intermittent presence of neighbors. Thus a given vehicle can communicate only periodically. For this density level the total of communicating periods is about $20 \%$ of the simulation time for each vehicle (during the remaining time, the vehicle is considered to be without any neighbor).

For these various density levels, the evolutionary algorithm returned a set of solutions (those in the first Pareto front) [13]. We then sorted the best solutions in order to select the one that offered the best balance between the reachability and saturation of the channel. These results are presented in Table 3.

The average propagation time as well as the average number of collisions only concerns the runs of simulations that have covered the whole study area (among the 500 runs). For very low density networks, the simple flooding and the other probabilistic methods are not applicable. They have almost zero probability of spreading a message to all the nodes over a distance of $10 \mathrm{~km}$. Thus, figures 3 to 5 provide no result for those methods in very sparse networks.

Figure 3 compares the considered broadcasting methods with respect to the full reception ratio. That is the probability for those techniques to ensure a complete coverage of the study area. In high density networks, the four methods ensure complete coverage of the study area. However, when the network density decreases, the quality of results provided by the Simple flooding and two probabilistic methods degrade. In such networks, ensuring a wide dissemination of messages depends on the number of times the relays repeat the packets. When this number is low (equal to 1 for example), packet reception ratio decreases gradually as they propagate and communication eventually stops. This phenomenon is particularly noticeable for networks with very low density. These results show that when a vehicle has about $20 \%$ of chance to have a neighbor that receives its packets, the message must be repeated twenty times (see Table 3).

For full coverage in low-density or very low-density networks, Smart-flooding advocates to resend packets several times. The protocol assumes that collisions or distance between the source and the destination may cause an interruption of the broadcast. The message will reach all nodes after a new issue. The histogram in Figure 4 illustrates this by the relatively high propagation delay of the Smart-flooding in the low density levels. We conclude that the total coverage 
of the network is at the expense of dissemination speed. In dense or very dense environments, the propagation time of the different broadcasting methods are equivalent. This reflects the fact that in such cases, the first front of broadcast $(N r=1)$ enables the dissemination of the emergency message throughout the study area. As expected, the number of collisions decreases with network density (Figure 5). The difference between the four broadcasting methods is slight. Even when Smart-flooding repeats packets more than once, it regulates the broadcast using good $P$ and $D r$ values.

\begin{tabular}{|l|l|l|}
\hline $\begin{array}{l}\text { Density } \\
\text { level }\end{array}$ & $\begin{array}{l}\text { Inter- } \\
\text { vehicle } \\
\text { distance }\end{array}$ & $\begin{array}{l}\text { Number } \\
\text { of nodes }\end{array}$ \\
\hline High & $25 \mathrm{~m}$ & 400 \\
\hline Medium & $75 \mathrm{~m}$ & 134 \\
\hline Low & $200 \mathrm{~m}$ & 50 \\
\hline
\end{tabular}

Table 2: Network densities

\begin{tabular}{|l|l|l|l|l|}
\hline Density & $P$ & $N r$ & $\begin{array}{l}D r \quad \text { (in } \\
\text { seconds) }\end{array}$ & TTL \\
\hline Very low & 0.9999852 & 21.0 & 0.5588954 & 20.0 \\
\hline Low & 0.9158122 & 2.0 & 0.7285401 & 28.0 \\
\hline Medium & 0.7755884 & 1.0 & 1.5412072 & 26.0 \\
\hline High & 0.3591064 & 2.0 & 0.6757185 & 18.0 \\
\hline
\end{tabular}

Table 3: Smart-flooding solutions

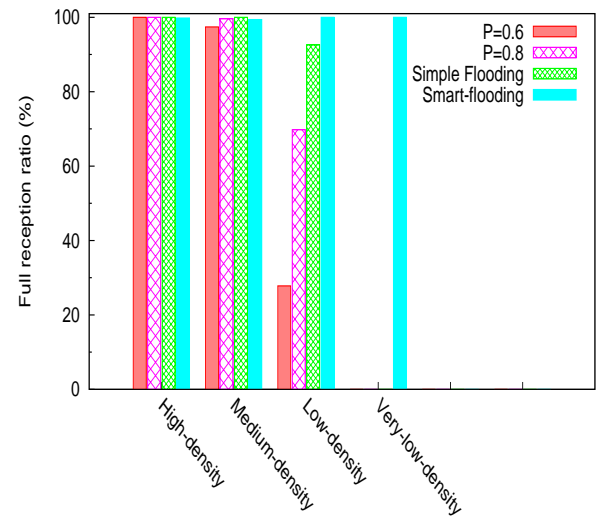

Neighborhood density

Fig. 3: Full Reception Ratio

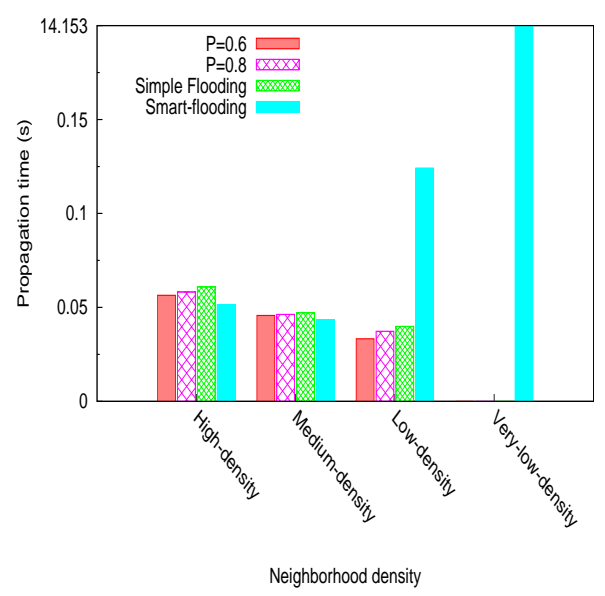

Fig. 4: Average propagation time

\subsection{Tests in a heterogeneous network}

The density of VANETs is not generally homogeneous. In a urban environment for example, the density is not the same at a downtown and on the outskirts of the city. The network topology depicted in Figure 7 is used to compare the four broadcasting methods. This network is composed of three main levels of density: low-density, medium-density and high density. 


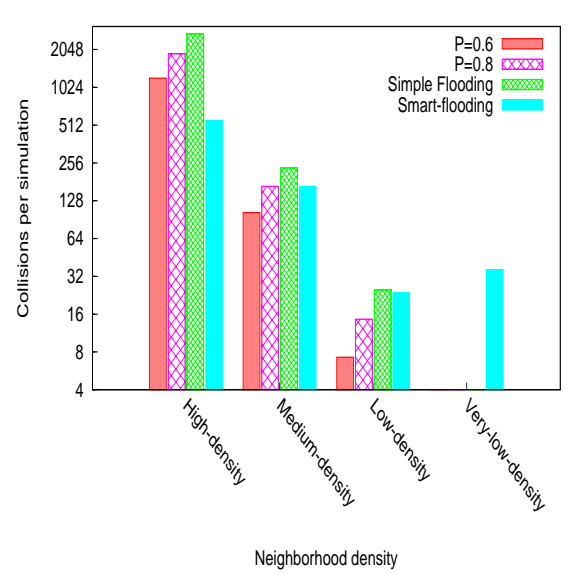

Fig. 5: Average number of collisions

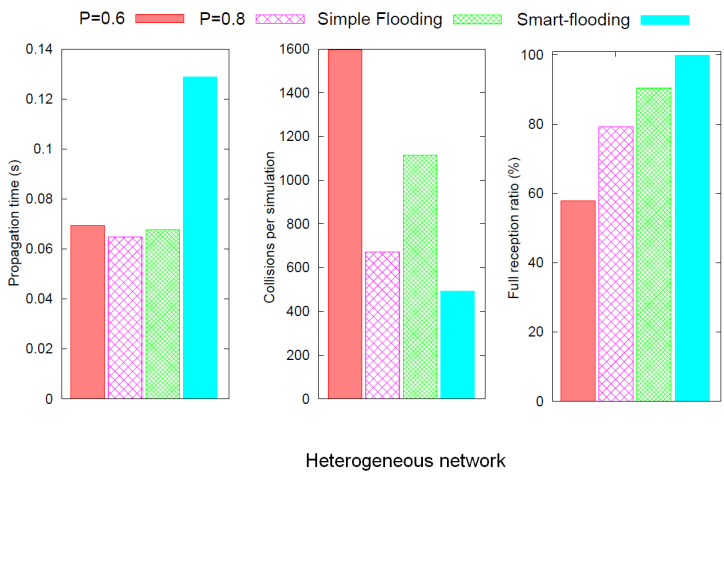

Fig. 6: Heterogeneous network results

The results of experiments in the heterogeneous network are shown in Figure 6 . The warning message is sent by the rightmost node of Figure 7 . The strategy used by Smart-flooding slightly slow down the spread of the message when it reaches the high density area to avoid a too high number of collisions. Collisions may prevent a wide dissemination of the message. Thus, Smart-flooding tolerates the first broadcasting front fail. It also allows nodes retransmit packets twice $(N r=2)$ in very dense environments. This redundancy allows wide dissemination of the message, even if it causes a slightly greater delay than the three other gossip methods (order of a few hundredths of seconds). Please note that this redundancy has no impact on the saturation of the channel since Smartflooding uses low probabilities for very high density network (see Table 3). This is also illustrated by a collision rate significantly lower than for other methods. When considering the third criterion of comparison, the simple flooding and the other two probabilistic methods do not provide full coverage of the network. Their coverage is between $57 \%$ and $90 \%$ whereas Smart-flooding covers the whole network. The purpose of this experiment was to evaluate the behavior of broadcasting protocols in a heterogeneous environment. The goal is to transmit the message quickly and to make it reach all nodes in the studied area. The slight difference of propagation time does not diminish the Smart-flooding results. The difference with the results of other protocols is only a few hundredths of seconds.

\section{5 conclusion}

A new broadcasting protocol for MANETs (Smart-flooding) is proposed in this paper. Its parameters are determined by an evolutionary algorithm. Smartflooding is compared to three other probabilistic methods on a sending emergency message problem in a VANET with low, medium and high densities. This 


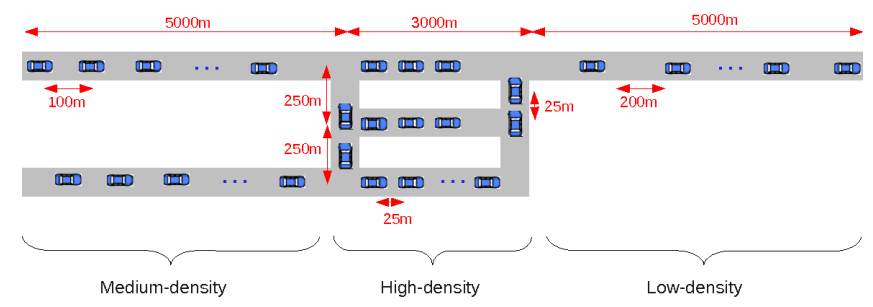

Fig. 7: Heterogeneous network topology

comparative study shows that Smart-flooding covers the whole network while other methods have a lower coverage probability (from $57 \%$ to $90 \%$ coverage) for the same network. The average number of collisions and the average time of propagation remain almost equivalent for the four methods, except that the propagation time increases to about 14 seconds in very low-density. But in this case, Smart-flooding manages to spread the warning message to all nodes, while other methods fail. The two main prospects of this work are to extend this protocol to dynamic multi-radio networks, and integrate a criterion of minimizing energy consumption in order to define the best compromise between performance and longevity of the network.

\section{Acknowledgement}

We thank Samuel Buendia for his participation in the project that led to the development of Vanet Data Representation (VDR) at the Computer Science Laboratory of the University of Franche-Comté.

\section{References}

1. E. Alba, B. Dorronsoro, F. Luna, A. J. Nebro, and P. Bouvry. A cellular multiobjective genetic algorithm for optimal broadcasting strategy in metropolitan manets. In IPDPS '05: Proceedings of the 19th IEEE International Parallel and Distributed Processing Symposium (IPDPS'05) - Workshop 6, page 192.1, Washington, DC, USA, 2005. IEEE Computer Society.

2. Dang Nguyen and Pascale Minet. Analysis of mpr selection in the olsr protocol. In AINAW '07: Proceedings of the 21st International Conference on Advanced Information Networking and Applications Workshops, pages 887-892, Washington, DC, USA, 2007. IEEE Computer Society.

3. N. Karthikeyan, V. Palanisamy, and K. Duraiswamy. Optimum density based model for probabilistic flooding protocol in mobile ad hoc network. European Journal of Scientific Research, 39(4):577-588, 2010.

4. Abdalla M. Hanashi, Aamir Siddique, Irfan Awan, and Mike Woodward. Dynamic probabilistic flooding performance evaluation of on-demand routing protocols in 
manets. In CISIS '08: Proceedings of the 2008 International Conference on Complex, Intelligent and Software Intensive Systems, pages 200-204, Washington, DC, USA, 2008. IEEE Computer Society.

5. Abdalla M. Hanashi, Irfan Awan, and Mike Woodward. Performance evaluation based on simulation of improving dynamic probabilistic flooding in manets. In WAINA '09: Proceedings of the 2009 International Conference on Advanced Information Networking and Applications Workshops, pages 458-463, Washington, DC, USA, 2009. IEEE Computer Society.

6. L. Li, J. Halpern, and Z. Haas. Gossip-based ad hoc routing. In Proceedings of the IEEE INFOCOM, IEEE Computer Society, 2002.

7. M. Bani Yassein, A. Al-Dubai, M. Ould Khaoua, and Omar M. Al-jarrah. New adaptive counter based broadcast using neighborhood information in manets. Parallel and Distributed Processing Symposium, International, 0:1-7, 2009.

8. Amin Vahdat and David Becker. Epidemic routing for partially-connected ad hoc networks. Technical report, 2000.

9. A. Lindgren and A. Doria. Probabilistic routing protocol for intermittently connected networks, internet draft. Technical Report draft-irtf-dtnrg-prophet, Internet Engineering Task Force, february 2010. http://tools.ietf.org/html/draft-irtf-dtnrgprophet.

10. T. Spyropoulos, K. Psounis, and C.S. Raghavendra. Single-copy routing in intermittently connected mobile networks. In IEEE Communications Society Conference on Sensor and Ad Hoc Communications and Networks, pages 235 - 244, 2004.

11. K. Fall and S. Farrell. DTN: an architectural retrospective. Selected Areas in Communications, IEEE Journal on, 26(5):828-836, 2008.

12. The Network Simulator Project - ns-2, http://www.isi.edu/nsnam/ns.

13. Wahabou Abdou, Adrien Henriet, Dominique Dhoutaut, François Spies, and Christelle Bloch. Optimizing communications in vehicular ad hoc networks using evolutionary computation and simulation. In CSTST'2008, Int. Conf. on Soft Computing as Transdisciplinary Science and Technology, pages 158-165, Cergy-Pontoise, France, 2008. ACM.

14. Wahabou Abdou, Adrien Henriet, Christelle Bloch, Dominique Dhoutaut, Damien Charlet, and François Spies. Using an evolutionary algorithm to optimize the broadcasting methods in mobile ad hoc networks. Journal of Network and Computer Applications, 2011.

15. Kalyanmoy Deb, Samir Agrawal, Amrit Pratap, and T. Meyarivan. A fast and elitist multiobjective genetic algorithm: Nsga-ii. IEEE Trans. Evolutionary Computation, 6(2):182-197, 2002.

16. Eckart Zitzler, Marco Laumanns, and Lothar Thiele. Spea2: Improving the strength pareto evolutionary algorithm. Technical report, Computer Engineering and Networks Laboratory (TIK),Swiss Federal Institute of Technology (ETH) Zurich, 2001.

17. Aleš Zamuda, Janez Brest, Borko Boškovic, and Viljem Žumer. Differential evolution with self-adaptation and local search for constrained multiobjective optimization. In CEC'09: Proceedings of the Eleventh conference on Congress on Evolutionary Computation, pages 195-202, Piscataway, NJ, USA, 2009. IEEE Press.

18. Qingfu Zhang, Aimin Zhou, Shizheng Zhao, Ponnuthurai Nagaratnam Suganthan, Wudong Liu, and Santosh Tiwari. Multiobjective optimization test instances for the cec 2009 special session and competition. Technical report, The School of Computer Science and Electronic Engieering, 2009. 
19. D. Dhoutaut, A. Regis, and F. Spies. Impact of radio propagation models in vehicular ad hoc networks simulations. In VANET'06: Procs of the 3rd int. workshop on Vehicular ad hoc networks, pages 40-49, Los Angeles, USA, 2006. ACM Press. 